A N N A L E S Annales de Bretagne et des Pays de l'Ouest

\title{
Anthropologie, Mythologies et histoire de la chevelure et de la pilosité
}

\section{Bernard Merdrignac}

\section{(2) OpenEdition}

1 Journals

\section{Édition électronique}

URL : http://journals.openedition.org/abpo/2332

DOI : $10.4000 / a b p o .2332$

ISBN : 978-2-7535-1852-0

ISSN : 2108-6443

\section{Éditeur}

Presses universitaires de Rennes

\section{Édition imprimée}

Date de publication : 30 mars 2012

Pagination : 189-192

ISBN : 978-2-7535-1850-6

ISSN : 0399-0826

\section{Référence électronique}

Bernard Merdrignac, "Anthropologie, Mythologies et histoire de la chevelure et de la pilosité », Annales de Bretagne et des Pays de l'Ouest [En ligne], 119-1 | 2012, mis en ligne le, consulté le 20 septembre 2020 URL : http://journals.openedition.org/abpo/2332 ; DOI : https://doi.org/10.4000/abpo.2332

Ce document a été généré automatiquement le 20 septembre 2020.

(c) Presses universitaires de Rennes 


\title{
Anthropologie, Mythologies et histoire de la chevelure et de la pilosité
}

\author{
Bernard Merdrignac
}

\section{RÉFÉRENCE}

Bertrand Lançon, Marie-Hélène Delavaud-Roux (dir.), Anthropologie, Mythologies et histoire de la chevelure et de la pilosité, Le sens du poil, Paris, L'Harmattan, 2011, 353 p. (ISBN 978-2-296-54553-3).

Cet ouvrage est issu d'un colloque transdisciplinaire (Histoire, sociologie, ethnologie, littérature, iconographie, médecine, philosophie, anthropologie, psychologie) organisé à la Faculté de lettres et sciences humaines Victor-Segalen de Brest en 2007 qui a permis de mettre en relation les points de vue des diverses sciences humaines et l'apport des discours littéraires sur « le poil ». Le sujet qui peut paraître de prime abord "tiré par les cheveux» comme l'écrit Bertrand Lançon en introduction n'a rien d'anecdotique. L'archéologie qui a « exhumé tant de peignes du passé » (sans parler des rasoirs ou des ciseaux) en dit long sur l'importance sociale de ce « jardinage corporel ». À travers les époques, et les sociétés, la pilosité est « sémiophore » (pour emprunter ce néologisme à Krzysztof Pomian). « Porteurs de signes sociaux », poils, cheveux, barbe et moustache sont l'expression d'identifications sexuelles, ethniques, religieuses, politiques ou philosophiques voire de relations à la bestialité et à l'humanité, à la sagesse et à la barbarie, à l'ascèse et à la sensualité. Le poil domestiqué, indicateur d'ordre, peut à l'inverse, dans le désordre, virer à l'incongruité voire au scandale. Il n'est pas indifférent que La Cantatrice chauve de Ionesco ait été la pièce la plus jouée de la seconde moitié du siècle dernier. Les responsables de l'édition de ces Actes ont choisi de distribuer les contributions rassemblées autour de six thèmes principaux que cet ouvrage collectif ne saurait évidemment, de leur point de vue, avoir l'ambition d'épuiser. Plutôt que de parties à proprement parler, il s'agit de dossiers qui interfèrent à l'occasion. Le premier de ceux-ci, consacré aux « mythologies pileuses et capillaires : figures emblématiques » est suivi d'un deuxième qui s'interroge sur « la place, les rôles 
et sens » de la pilosité avant qu'une troisième série de communications n'aborde la question de «l'ablation; du sacrifice à la punition ». Un quatrième dossier qui s'attache à dégager les "alternatives pileuses" se concentre sur le binôme "masculinité/ féminité » (que l'on retrouve bien entendu par ailleurs en arrière-plan de plusieurs autres thèmes), tandis que le cinquième évoque les «représentations: images littéraires, picturales, cinématographiques». Enfin cette répartition thématique se boucle (si l'on peut dire) par quelques réflexions sur «ordre et désordre: société politique, police».

2 Sinon par manque de curiosité, du moins par défaut de compétences interdisciplinaires, le signataire du présent compte-rendu n'aura pas non plus la prétention de passer en revue chacune de ces vingt-deux contributions. Il doit donc se contenter de proposer quelques ouvertures sur les multiples perspectives offertes par le livre avant de mettre en relief certaines communications susceptibles de retenir particulièrement l'intérêt des lecteurs des Annales de Bretagne et des Pays de l'Ouest. Parmi les pépites que l'on découvre au fil de la lecture, la communication de Gérard Moitrieux (« la barbe dans la sculpture gallo-romaine ») ouvre brillamment le feu en posant d'emblée la question qui tue : « la barbe fait-elle le gaulois? », à partir des statues d'Hercule et d'autres divinités barbues, généralement considérées comme identitaires. Or l'analyse des représentations d'une divinité incontestablement celtique comme Cernunnos démontre que la barbe ne saurait être le signe de l'appartenance au substrat celtique. Si le dieu est effectivement parfois barbu comme à Paris ou à Reims, il est tout aussi souvent imberbe comme à Metz ou à Vendeuvres et surtout sur le célèbre chaudron de Gundestrup. Pour sa part, Pierre Brulé rappelle d'abord opportunément que l'offrande de la pilosité est une pratique rituelle généralisée (et de ce fait polysémique) dans le monde grec. C'est pourquoi son étude intitulée «Dans des épigrammes de l'Anthologie grecque, offrir sa chevelure aux dieux » se cantonne à l'oblation rituelle de la chevelure à la puberté pour les garçons et à son équivalent pour les filles au moment du mariage. L'acte permet donc aux plus jeunes de croître en beauté et aux filles de connaître une union et une maternité favorables. Dans un tout autre registre, la contribution d'Ivan Ricordel (directeur du Laboratoire de toxicologie de la Préfecture de Police de Paris), "L'analyse des cheveux et des poils dans les enquêtes criminelles" fait un point fort utile sur l'apport de l'analyse toxicologique de l'ADN «possiblement présent dans les cheveux » en matière de crime par empoisonnement. On appréciera les mises en garde de ce spécialiste contre des conclusions trop hâtives, illustrées (entre autres) par l'exemple de la présence d'arsenic dans les mèches de cheveux de Napoléon qui se retrouve de temps à autre dans la presse grand public. Parmi les diverses hypothèses envisageables, celle de l'intoxication n'est ni la seule à pouvoir être retenue, ni même la plus plausible. C'est pourquoi l'étude de cas par Peter Hvilshöj Andersen sur « Les vieux cheveux renfermant la clef d'Hamlet" paraît devoir être prise avec précaution. L'explication traditionnelle du décès en 1601 de l'astronome danois telle que la rapporte encore Milan Kundera en 1990 dans l'Immortalité est certes invraisemblable. $\mathrm{Au}$ cours d'un dîner officiel durant son exil à Prague «[...] il refréna pudiquement son envie d'aller aux cabinets si bien que sa vessie éclata, et lui, martyr de la honte et de l'urine, s'en fut promptement rejoindre les immortels risibles"! Par contre, de récentes analyses toxicologiques de poils de barbe puis de cheveux de Tycho Brahé pourraient donner crédit à des rumeurs qui, dès le xvII e siècle, le disaient victime d'un empoisonnement au mercure. Dont acte. Cependant l'hypothèse avancée ici selon laquelle l'instigateur du crime aurait été le roi Christian IV de Danemark (ce que 
confirmerait un décryptage du drame de Shakespeare Hamlet) semble relever davantage du Da Vinci Code que de l'histoire. Au contraire, l'historicité de Vlad Tepes, le voïvode valaque $\mathrm{du} \mathrm{xv}^{\mathrm{e}}$ siècle qui serait le probable prototype du personnage de Dracula dans le roman "néogothique » de Bram Stoker ne retient pas l'attention de Solène Robert (" Du poil de la bête »). Celle-ci s'attache plutôt à montrer comment à la croisée de données médicales (la porphyrie se manifeste, entre autres, par une hyperpilosité) et des croyances populaires relatives aux revenants, vampires et autres garous (recueillies au XvIII ${ }^{\mathrm{e}}$ siècle par le polygraphe mauriste dom Calmet ; sur cet érudit mauriste, voir le compte-rendu par Marie Berthelot de Philippe MARTIN et Fabienne HENRYOT [dir.], Dom Augustin Calmet. Un itinéraire intellectuel, Paris, éditions Riveneuve, 2008, dans ABPO, 2011/1, p. 201-203), le vampire imaginé par l'auteur anglo-irlandais est d'abord velu et animal, avec notamment «des poils au milieu des paumes». Repris par le cinéma, le vampire s'humanise progressivement et perd ses poils jusqu'à devenir le personnage séducteur et sensuel incarné par Christopher Lee. En définitive, il ne reste que les dents pour évoquer la bestialité du monstre assoiffé de sang.

3 En esquissant une subtile comparaison entre le succès de Dracula et celui du Roman de Renart « où le goupil est devenu renard ", cet article de S. Robert fait penser au chapitre de Michel Pastoureau sur «L'homme roux. Iconographie médiévale de Judas» (Une histoire symbolique du Moyen Age occidental, Paris, Seuil, 2004) qui n'a pas échappé à l'érudition impeccable de Jean-Christophe Cassard dans sa contribution sur laquelle on reviendra ci-dessous. Le roux n'est-il pas, pour M. Pastoureau, « la couleur des démons, du goupil, de l'hypocrisie, du mensonge et de la trahison»? David Lavergne («Pyrrhos, gloire et péchés d'un roux») souligne ici les connotations de la "couleur rouge", incarnant les excès de la fonction guerrière dans la littérature antique, tout en soulignant les affinités de Pyrrhos avec «Esaü le velu» dont Benoît Jeanjean fait «la figure biblique du réprouvé chez les Pères de l'Eglise ». Dépossédé selon la Genèse de son droit d'aînesse contre un plat de lentilles par suite de la ruse de Jacob, Esaü figure pourtant le pécheur, le juif, l'hérétique chez Augustin et Jérôme. L'auteur ne signale qu'en passant la rousseur du personnage, mais fait ressortir pertinemment toute l'ambivalence du poil dans la Bible. Il n'est dévalorisé par l'exégèse qu'en opposition avec la « peau lisse » de Jacob. Il s'agit ici du poil des bêtes sauvages! Sans doute auraitil été possible d'insister davantage encore sur la contradiction entre le modèle du chasseur et celui du pasteur qu'incarnent ces deux figures. On retrouve en effet des échos de celle-ci dans plusieurs communications relatives au haut Moyen Âge telles que l'étude de Bruno Dumézil sur la "décalvation » dans les législations wisigothiques ou celle de Magali Coumert sur «la pilosité barbare dans les premiers siècles du Moyen Âge occidental». L'article s'ouvre sur la description par Amien Marcelin de la "chevelure teinte en rouge» des Alamans destinée à souligner la «terrifiante sauvagerie du barbare ». L'auteur relève qu'à la fin du vie siècle, Grégoire de Tours «note que les Bretons se distinguaient par la coupe et le costume, mais ne les décrit jamais ».

4 C'est probablement pourquoi la Bretagne se voit attribuer la portion congrue dans le présent ouvrage. On peut néanmoins (et sans doute le doit-on?) tirer un coup de chapeau à Sabine Dutray («La symbolique du cheveu au cours de l'histoire de la Bretagne. Le cheveu, support d'expression symbolique en Bretagne : représentations celtes et bretonnes»). Nourrie de quelques lectures (mal digérées?) d'ouvrages généraux sur la Gaule (Henri Hubert, Christian-Jean Guyonvarc'h, Barry Cunliffe) et sur 
la Bretagne moderne et contemporaine (Paul Sébillot, Ernest Renan, René-Yves Creston, Jean-Marie Ploneis, Ronan Le Coadic) accommodées à la sauce psychanalytique, elle réussit à accumuler en une dizaine de pages une somme d'interprétations décoiffantes. Le lecteur intéressé par ce type d'approche fera bien mieux de se reporter aux études comparatistes, solidement documentées, du professeur Claude Sterckx sur la mythologie celtique. Sinon, il sera peut-être ébahi de découvrir ici que le chapeau breton ne serait rien de moins qu'un «un phallus acceptable, "politiquement correct" " et que la "règle surmoïque » imposée par les recteurs et symbolisée par la coiffe féminine vise à réduire cette "pulsion potentiellement fascinante » de la chevelure «à l'impuissance totale » [...] « par répression complète de la libido" On aurait presque envie de qualifier ces spéculations aventureuses de désopilantes si l'étymologie le permettait. Mais ce n'est pas possible; par contre, elle n'interdit pas de les traiter d'horripilantes.

5 L'auteure prend cependant la précaution de préciser que ses propos ne sont peut-être que le fruit des projections de sa "propre histoire» (qui ne concerne pas forcément tout le monde ?) et concède « [...] avoir entendu depuis l'enfance que la Bretagne était un pays mystérieux et les Bretons des hommes pas comme les autres». Fort heureusement, J.-C. Cassard est là pour nous rassurer. À l'encontre de la psychologue clinicienne qui se contente avec désinvolture de moins d'une quinzaine de notes et saute allègrement par-dessus le millénaire médiéval, la brillante synthèse de cet historien du Moyen Âge s'appuie sur soixante-cinq références renvoyant précisément aux recherches récentes sur les sources textuelles, iconographiques, onomastiques, etc. Il établit que " capillairement corrects les Bretons médiévaux » ne se distinguent guère "de ce que l'on trouverait dans d'autres régions de France et d'Europe ». On relèvera, par exemple, que la fameuse "tonsure celtique», soi-disant d'origine druidique, "d'une oreille à l'autre » (ab aure usque ad aurem), que l'on imagine en général, comme épargnant la chevelure à l'arrière (seul le devant de la tête aurait été rasé), a été virtuellement reconstituée en trois dimensions par les chercheurs irlandais à partir des enluminures du haut Moyen Âge (Daniel MCCARTHY, "On the Shape of the Insular Tonsure », Celtica, 24, 2003, p. 140-167). Ajoutons que sa forme de triangle isocèle (l'angle supérieur au milieu du front et la base au sommet du crâne) expliquerait l'énigmatique sobriquet d'asciciput ("tête de hache») appliqué par ses adversaires à saint Patrice d'après sa Vie tripartite. On retiendra encore que, vers la fin du Moyen Âge, les codes de la bienséance française sont de rigueur dans le duché : la barbe est signe de sagesse ; la chevelure drue témoigne de la vitalité de l'adulte; la tonsure soignée marque la retenue qui sied au clergé. Mais l'anglophile Jean IV se distingue par sa moustache à l'anglaise comme celle de son beau-père le Prince noir. "Comme quoi le poil n'est pas tout à fait étranger aux querelles politiques du temps!» Pour conclure, il est réjouissant de constater que dans l'imaginaire actuel (tel que l'exprime la bande dessinée) le Moyen Âge breton est " automatiquement à poil long et à barbe ", comme le montre le «Dogue noir de Brocéliande » de Gilles Chaillet. L'animalité a encore de beaux jours devant elle! 